\title{
BMJ Open Natural history of glaucomatous optic neuropathy in highly myopic Chinese: study protocol for a registry cohort study
}

\author{
Yunhe Song, ${ }^{1}$ Wei Wang (D) , ${ }^{1}$ Fengbin Lin, ${ }^{1}$ Shida Chen, ${ }^{1}$ Ling Jin, ${ }^{1}$ Fei Li, ${ }^{1}$ \\ Kai Gao, ${ }^{1}$ Weijing Cheng, ${ }^{1}$ Jian Xiong, ${ }^{1}$ Rouxi Zhou, ${ }^{1}$ Meiling Chen, ${ }^{1}$ Jiaen Liang, ${ }^{1}$ \\ Jiani Zhang, ${ }^{1}$ Jost B Jonas (D) , ${ }^{2}$ Xiulan Zhang (D) ${ }^{1}$
}

To cite: Song Y, Wang W, Lin F, et al. Natural history of glaucomatous optic neuropathy in highly myopic Chinese: study protocol for a registry cohort study. BMJ Open 2020;10:e039183. doi:10.1136/ bmjopen-2020-039183

- Prepublication history for this paper is available online To view these files, please visit the journal online (http://dx.doi org/10.1136/bmjopen-2020039183).

Received 08 April 2020

Revised 10 November 2020

Accepted 18 November 2020
Check for updates

(c) Author(s) (or their employer(s)) 2020. Re-use permitted under CC BY-NC. No commercial re-use. See rights and permissions. Published by BMJ.

\section{${ }^{1}$ State Key Laboratory of} Ophthalmology, Zhongshan Ophthalmic Center, Sun Yat-sen University, Guangzhou, China ${ }^{2}$ Department of Ophthalmology, Medical Faculty Mannheim, Heidelberg University, Heidelberg, Baden-Württemberg, Germany

Correspondence to Dr Xiulan Zhang; zhangx|2@mail.sysu.edu.cn

\section{ABSTRACT}

Introduction Myopic maculopathy and glaucoma belong to the most common causes of irreversible blindness worldwide and, having an ocular axial elongation as one of their main risk factors, can occur together. The detection of glaucomatous optic neuropathy (GON) in highly myopic eyes is clinically and technically difficult, and there is no information available, neither about the natural course of GON or about the course of GON under intraocular pressure-lowering therapy. We therefore designed this study to explore the natural course of GON in highly myopic eyes.

Methods and analysis In this single-centred longitudinal registry cohort study, 813 highly myopic individuals will be recruited and undergo detailed ophthalmic examinations. High myopia is defined by a myopic refractive error of $\geq-6$ D or an axial length of $\geq 26.5 \mathrm{~mm}$. GON is defined by a glaucomatous appearance of the optic nerve head or glaucomatous visual field (VF) defects. GON progression is defined by either change of the optic disc or VF.

Ethics and dissemination Ethical approval has been obtained from the ethical committee of the Zhongshan Ophthalmic Center (ZOC), Sun Yat-sen University, China (ID: 2019KYPJ079). All the participants are required to provide informed consents. Results will be disseminated through scientific meetings and published in peer-reviewed journals. The data will be deposited at the clinical research centre in ZOC using electronic data capture system, and a copy of paper files will also be kept. Only members of the project team will have access to these data.

Trial registration number NCT04302220.

\section{INTRODUCTION}

Recent meta-analyses of available epidemiological data have revealed that glaucoma and myopic maculopathy (MMD) belong to the most common causes of irreversible blindness worldwide. ${ }^{1}$ The main risk factor for MMD is axial elongation, which also is an important risk factor for the prevalence of glaucomatous optic neuropathy $(\mathrm{GON}) .^{2} 3$ Consequently, GON and MMD can occur together, with the GON prevalence increasing with

\section{Strengths and limitations of this study}

- The study will illustrate the distribution of glaucomatous optic neuropathy (GON) in a highly myopic Chinese population.

- Structural and functional changes of glaucomatous optic neuropathy will be analysed and compared comprehensively in highly myopic eyes during longterm follow-up.

- Multiple objective and subjective assessment will be carried out of ocular parameters in all participants.

- Since the natural course of the GON in highly myopic eyes is unknown, extend follow-up longer than 3 years if necessary.

- The sampling is not population-based, and the generalisation will be limited.

longer axial length in the highly myopic eyes. A recent hospital-based study demonstrated that the prevalence of GON was $27.2 \%$ in highly myopic eyes (mean axial length of $29.5 \pm 2.2 \mathrm{~mm}$ ). Moreover, in subgroup analysis, as the mean axial length grew from $<26.5 \mathrm{~mm}$ to $>28 \mathrm{~mm}$, the prevalence grew from $12.2 \%$ to $32.6 \%$, and there were $42.1 \%$ of subjects having GON with the axial length $\geq 30 \mathrm{~mm}^{4}$

It is difficulty to detect the glaucomatous neuropathy in high myopia both in clinical practice and in research. The classic inferior-superior-nasal-temporal rule was not satisfied in assessment of optic nerve head due to the deformation of the optic nerve head in highly myopic eyes particularly in intrapapillary vessel kinking relatively near to disc border. ${ }^{5}$ In clinical diagnosis, myopiarelated optical nerve defomation can hamper the judgement of the size, shape and colour of the optic disc. ${ }^{6}$ There were several aspects: (1) reduction of the spatial contrast by comparing the neuroretinal rim height and 
the optic cup depth that is may be due to the lamina cribrosa stretching or optic cup flattening; (2) decrease in the colour contrast by comparing the neuroretinal rim and the bottom of the optic cup, which were presented as relatively pink and pale, repectively; (3) visibility reduction of the peripapillary retinal nerve fibre layer thickness caused by a relatively lighter background in gamma zone and delta zone; (4) technical difficulties in the measurement of the peripapillary retinal nerve fibre layer thickness using optical coherence tomography; (5) atypical visual field defects that could be due to myopic macular degeneration, refractive errors, deformation of the posterior eye wall (eg, due to staphylomas) or an optic nerve damage. In addition, the intraocular pressure (IOP) is often within the normal range in highly myopic eyes with GON. ${ }^{7}$ In addition to other methods, recent studies have also explored the possibility of measuring the vascular density in the parapapillary region as a substitution for the retinal nerve fibre layer thickness and the possibility of measuring the thickness of the ganglion cell-inner plexiform layer in the the macular region. ${ }^{8-11}$ The clinical practicability of these techniques has however not been fully proven for the detection of GON in highly myopic eyes.

The difficulties in the detection of the presence and progression of GON in highly myopic eyes were some of the reason why there is almost no information available, neither about the natural course of GON nor about the course of GON under intraocular pressure (IOP)lowering therapy. In contrast to the occurrence of GON in highly myopic eyes, the prevalence of MMD and its associated factors have been studied in greater detail, while information on longitudinal changes in MMD have remained to be scarse so far. ${ }^{12}{ }^{13}$ The META analysis for Pathologic Myopia (META-PM) Study Group consortium formulated guidelines to categorise the stage of MMD in highly mypic eyes. ${ }^{14}$ We therefore designed this study to explore the natural course of GON in highly myopic eyes as well as to observe morphological non-glaucomatous changes occurring in highly myopic eyes. The objectives are to investigate the distribution and structural and functional changes of GON in highly myopic Chinese and to detect risk factors influencing the natural course of GON in highly myopic eyes.

\section{METHODS AND ANALYSIS}

The single-centred registry cohort study will be conducted at the Zhongshan Ophthalmic Center (ZOC), Sun Yatsen University, a tertiary hospital located at Guangzhou, Guangdong, China. All examinations will be carried out at the clinical researcg centre of the ZOC. Inclusion criterion is the presence of high myopia, defined by a myopic refractive error of $\geq-6 \mathrm{D}$ or an axial length $\geq 26.5 \mathrm{~mm}$. Other incolusion criteria and exclusion criteria are listed inbox 1.

This study protocol including the final version of the Patient Information and Consent Form was approved by

\section{Box 1 Inclusion and exclusion criteria}

Inclusion criteria:

- Age above 18 years.

- Diagnosed with high myopia.

- BCVA $\geq 6 / 12$.

Exclusion criterion:

- Unwilling or unable to give consent or unable to return for scheduled protocol visits.

- Glaucoma secondary to penetrating keratoplasty, trauma, steroids, retinal disease/surgery or neovascular disease.

- Obvious corneal and iris lesions or severe cataracts interfering with fundus examinations VF test or monophthalmia.

- Need for ocular surgery/laser or anticipated need for cataract surgery during the study period.

- Other serious systemic diseases (ie, hypertension, heart disease, diabetes or rheumatic immune system diseases).

- Pregnant or nursing women.

BCVA, best corrected visual acuity; IOP, intraocularpressure; VF, visual field.

the ethical committee of ZOC, Sun Yat-sen University, in April 2019 (ID: 2019KYPJ079).

The necessary sample size was calculated based on the primary outcome, that is, the onset and progression of GON during a follow-up period of 36 months. We took into account an odd ratio of 5.90 of the occurrence of GON in highly myopic eyes as compared with non-highly myopic eyes ${ }^{15}$ and an overall prevalence of primary openangle glaucoma of about $1.50 \% .{ }^{16}$ Assuming conservatively that the accumulative progression rate among high myopia is approximately $1.5 \% \times 5.90 \approx 8.80 \%$, according to the Beijing Eye Study, ${ }^{2}$ the cumulative incidence of GON is estimated to be $15 \%$ at a 3-year follow-up, and the standardised rate of onset and progression of GON in highly myopic eyes has been estimated to be around $12 \%$. At the two-sided significance level of 0.05 , a sample size of 650 subjects will be required using $95 \%$ confidence limits with an interval width of 0.05 . Estimating an attrition rate of $20 \%$ during the 36 months period, the final adjusted sample size is 813 . The sample size is calculated by PASS V.16.0 software (NCSs, LLC, Kaysville, Utah, USA).

The study will thus recruit 813 highly myopic subjects with the recruitment started in June 2019 and continued until June 2021. The study participants will be re-examined every 6 months. The flow chart of the study is listed in figure 1.

We define GON in highly myopic eyes either by the glaucomatous optic nerve head appearance or by VF defect using stereoscopic fundus photography and perimetric examination, respectively, as described in the Beijing Eye Study. Additionally, we choose a functional parameter (VF) and a structural parameter (morphology of optic disc), which were the two key factors for diagnosing and monitoring progression of GON. ${ }^{16}{ }^{17}$ Eyes with one of the following criteria were defined as GON with high myopia:

1. Myopic optic disc change mimicking glaucoma: In the definition of 'optic disc glaucoma', the only criterion for glaucoma was a glaucomatous appearance of the 


\section{Patients with}

high myopia
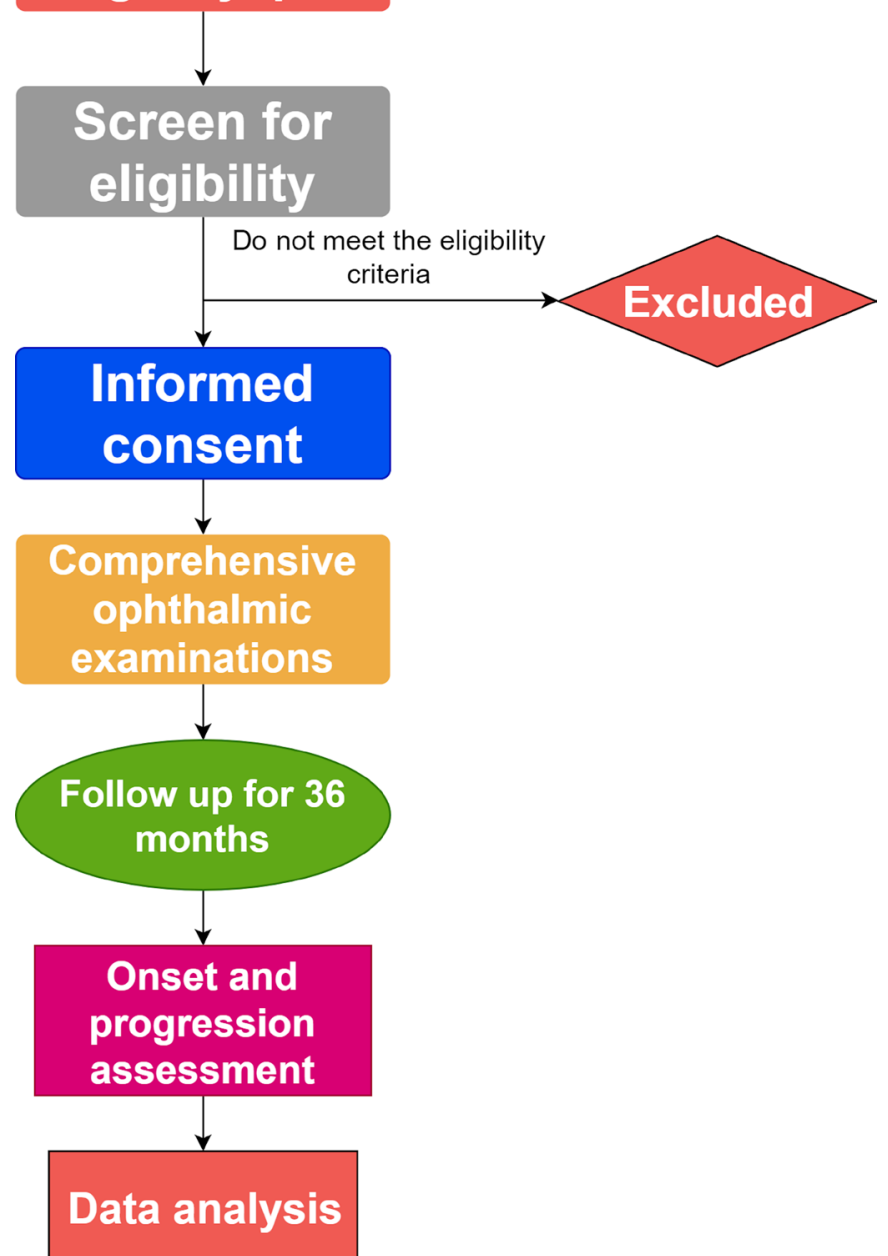

Figure 1 Flow chart of the study.

optic disc as described by $\mathrm{Xu} e t a l^{2}{ }^{2}$ The criteria were cited as follows:

Absolute criteria for a glaucomatous appearance of the optic nerve head, each of which were sufficient for the diagnosis of glaucoma, were a notch in the neuroretinal rim in the temporal inferior region and/or the temporal superior region, so that the inferior-superior-nasal-temporal rule was not fulfilled (in eyes with an optic cup sufficiently large to allow an assessment of the neuroretinal shape); a localized retinal nerve layer defect which could not be explained by any other cause than glaucoma; and an abnormally large cup in relation to the size of the optic disc. Relative criteria for the diagnosis of a glaucomatous appearance of the optic nerve head were if the neuroretinal rim was markedly thinner in inferior disc region compared with the superior disc region, even if the smallest part of the neuroretinal rim was located in the temporal horizontal disc region; a diffuse decrease in the visibility of the retinal nerve fiber layer (particularly in eyes with small optic discs), if the background pigmentation of the eye allowed an assessment of the retinal nerve fiber layer and if there were no other reasons than glaucoma for retinal nerve fiber layer loss; a marked diffuse thinning and/or focal thinning of the retinal arteries if there were no other reasons than glaucoma for retinal vessel thinning; and an optic disc hemorrhage, if there were no other reasons for a disc bleeding, such as retinal vessel occlusions. If none of the absolute glaucoma criteria were positive, at least 2 relative criteria had to be positive, including a suspicious neuroretinal rim shape in eyes with an optic cup large enough for the assessment of the rim shape; or at least 2 relative criteria had to be positive including the occurrence of an optic cup in a small optic disc which usually would not show cupping

2. Myopic eyes with glaucomatous VF defect: The glaucomatous VF defect is defined as a reproducible (at least two consecutive reliable tests) reduction in light threshold differential sensitivity at two or more contiguous points with a probability indicated by a $\mathrm{p}<0.01$ or more or three or more contiguous points with $\mathrm{p}<0.05$ or more in the pattern deviation plot, in the superior or inferior arcuate areas, or a $10 \mathrm{~dB}$ difference across the nasal horizontal midline at two or more adjacent points in the total deviation plot. The categorisation and grading of $\mathrm{VF}$ defects are referred as Zhongshan Ophthalmic Center-Brien Holden Vision Institute High Myopia Registry Study. ${ }^{18}$ The abnormal VF included the caracteristics of nasal steps, early arcuate scotomas, advanced arcuate scotomas, generalised reduction in the light threshold differential sensitivity, paracentral defects, central defects, tilted disc defect and cecocentral defect. ${ }^{18}$

The new devlopment or progression of GON is based on the perimetric results and the results obtained in the examination of the fundus photographs performed by two independent specialists in image reading. In case of a discrepancy between both reviewers, a panel will decide. If a participant shows a new development or progression of GON, the individual has reached the study endpoint but will continue in the study until the final visit.

\section{Patient and public involvement}

The study participants will be recruited when the protocol was approved. The patients were not invloved in the design, recruiment and development of research questions and outcome measures, which had become common and well-established clinical routines for all examinations. The subjects will be monitered closely and regularly and will be well informed before the informed consent is signed. The potential benefits and risks will be explained. The examination results will be presented at every visit during the follow-up to the study participants. The scientific results of the study will be published in peer-reviewed articles, without disclosing private information of the study participants. 


\section{Outcome measurement}

\section{Primary outcome}

The primary outcome is the onset and progression of GON within 36 months. The progression is defined as follows:

1. VF progression: Progression analysis is performed using the Humphrey Field Analyzer Mark 3 Guided Progression Analysis software and defined by at least three test points showing a significant negative change $(p<0.05)$ at the same locations ${ }^{19}$ compared to the baseline examination in two consecutive tests by the 24-2 VF program (tentative deterioration) and two confirmation texts leading to the same results. The confirmation examinations will be performed within 1 month. The first of two confirmation VFs is termed the 'progress point'.

2. Optic disc progression: Optic disc progression will be assessed by the comparison of the stereo optic nerve head photographs taken at baseline and at the followup examination. It is defined as any of the following ${ }^{20}$ :

(1) enlargement of vertical cup-to-disc ratio, (2) neuroretinal rim thinning, (3) wedge-shaped retinal nerve fiber layer defects (incidence or enlargement), (4) disc haemorrhage and (5) change in vessel position on the disc, if not related to myopic change.

\section{Secondary outcome}

1. Myopia progression: Change of best-corrected visual acuity after years. Axial elongation and spherical equivalence (SE) decrease will be collected respectively. Additionally, using fundus photos, myopic fundus changes will be observed and sorted. The progression of myopia is defined as an axial elongation by $0.1 \mathrm{~mm}$ or more over a year, the reduction of SE by $0.5 \mathrm{D}$ and a change in the classification of myopic maculopathy within the study period of 3 years.

2. Other structural changes of the retina and choroid using optical coherence tomography (OCT): Ganglion cell-inner plexiform layer (GC-IPL) thickness, choroid thickness and OCT angiography parameters.

\section{Investigations}

All examinations will be carried out at the clinical research centre in the ZOC. Experienced and trained technicians will perform inspection items for participants, during which the quality of the results will be supervised.

\section{Questionnaires}

The demographic information and the family and medical history will be obtained through standardised questionnaires by experienced clinical research coordinator (CRC). The demographic information include age (date of birth), gender, highest attained level of education and hobbies. The family history includes whether immediate family members within three generations have high myopia or glaucoma, as well as other ocular diseases. Medical histories are systematic disorders that potentially affect the progression of any ocular diseases.

\section{Slit-lamp biomicroscopy}

Examination of the anterior segment will be evaluated using a slit lamp (BQ-900, Haag Streit, Switzerland). The fundus check will be carried out using a $90 \mathrm{D}$ indirect ophthalmoscopy lens (Ocular 90D Slit Lamp Lenses, Ocular, Washington, USA).

\section{Refractometry SE and BCVA}

Using an auto refractometer (KR-800, Topcon, Japan), three measurements of the refractive error will be obtained for each eye, and the average of the values will be recorded. Using ETDRS LogMAR Chart (Precision Vision, Villa Prak, Illinois, USA) with standard illumination at a distance of $4 \mathrm{~m}$, experienced optometrists will perform subjective refractometry to measure the BVCA for every study participant.

\begin{tabular}{|c|c|c|c|c|c|c|c|c|c|c|}
\hline Items & Screening & Baseline & $6 M$ & $12 \mathrm{M}$ & $18 \mathrm{M}$ & $24 M$ & $27 M$ & $30 \mathrm{M}$ & $33 M$ & 36M EOS \\
\hline Informed consent & $x$ & $x$ & & & & & & & & \\
\hline Slit-lamp biomicroscopy & & $x$ & $x$ & $x$ & $x$ & $x$ & $x$ & $x$ & $x$ & $x$ \\
\hline Refraction, SE and BCVA & $x$ & $x$ & $x$ & $x$ & $x$ & $x$ & $x$ & $x$ & $x$ & $x$ \\
\hline OCT & & $x$ & $x$ & $x$ & $x$ & $x$ & $x$ & $x$ & $x$ & $x$ \\
\hline VF & & $x$ & $x$ & $x$ & $x$ & $x$ & $x$ & $x$ & $x$ & $x$ \\
\hline IOP & & $x$ & $x$ & $x$ & $x$ & $x$ & $x$ & $x$ & $x$ & $x$ \\
\hline $\mathrm{AL}$ and CCT measurement & & $x$ & $x$ & $x$ & $x$ & $x$ & $x$ & $x$ & $x$ & $x$ \\
\hline
\end{tabular}

$A E$, adverse event; AL, axial length; BCVA, best-corrected visual acuity; CCT, central corneal thickness; EOS, the end of the study; IOP, intraocular pressure; OCT, optical coherence tomography; SE, spherical equivalence; VF, visual field; $x$, examination for the eyes. 


\section{Fundus photography}

Two images centred on the disc (taken both under standardised stereoscopic conditions and under nonstandardised conditions) and a single image centred on the macular will be taken for each eye after dilation using stereo camera (Nonmyd WX3D, Kowa, Nagoya, Japan).

\section{Optical coherence tomography}

All patients will undergo a set of standardised OCT examinations using the default model centred on optic disc and macula using a swept-source device (DRI OCT Triton, TOPCON, Japan). The image quality should be higher than 60. In addition, a spectral domain OCT will be used to obtain measurements of the peripapillary retinal nerve fibre layer and the macular ganglion cell-inner plexiform layer thickness.

\section{VF examination}

All examinations will be performed with the Humphrey Field Analyzer Mark 3 (Carl Zeiss Meditec, Dublin, California, USA) and Swedish interactive threshold algorithm standard 24-2 program. The definition of different visual filed defects refers to a published article. ${ }^{15}$

\section{Intraocular pressure (IOP)}

Goldmann applanation tonometry (AT900, Haag Streit, Koeniz, Switzerland) will be used to measure the IOP, collected between 9:00 and 11:00 or between 14:00 and 16:00 at every study visit. The mean of the three measurement values will assessed and recorded. The time of measurement will also be recorded. The IOP will be remeasured at the same day and time as at the baseline examination to reduce the effect of diurnal IOP fluctuations.

\section{Ocular biometry}

The ocular biometric parameters will be measured by an optical biometry (IOLMaster 700, Carl Zeiss Meditec, Jena, Germany). It includes determination of the anterior chamber depth, central corneal thickness, lens thickness and axial length.

\section{Anthropometry and blood pressure}

The height and weight of the participants will be collected without shoes using a free-standing height rod and a calibrated scale (RGZ120, Jiangsu Wujin Weighing Apparatus Factory, Jiangsu, China). At the baseline visit, blood pressure is recorded at the right arm with the individual seated for at least $5 \mathrm{~min}$ (Omron M7 Blood Pressure Monitor (Matsusaka, Mie, Japan)).

\section{Measurement domains and follow-up time points}

The schedule of investigations is given in table 1. A total of nine visits within a 36-month period are planned, with the follow-up examinations being carried out every 6 months.

\section{Intervention}

This is a cohort study, and initial interventions will not be performed at the beginning of the study. If during the follow-up a participant shows progression of glaucoma, after consultation with two glaucoma experts (XZ and JBJ), a topical antiglaucomatous medication will be started. Appropriate treatment and timely referral to the retinal specialist will be provided to patients with a retinal disorder such as retinal detachment, macular degeneration or retinal neovascularisation.

\section{Data analysis}

The data will be presented as mean \pm SD for continuous variables with a normal distribution or as median (IQR) for non-normally distributed continuous variables and frequency (percentage) for categorical variables.

The risk factors associated with the development of GON (onset or progression) will be assessed statistically by performing logistic regression analyses, and the OR and their $95 \%$ CIs will be calculated. The variables with a $p$ value of less than 0.20 in the univariate regression analysis will be included into the multiple regression analysis. The longitudinal change across time will be assessed also for continuous secondary outcomes such as the GC-IPL layer thickness, choroidal thickness, OCT angiographic parameters, using mixed-effect models with restricted maximum likelihood estimation and unstructured variance-covariance structure. The Statistical Analysis Software V.9.4 (SAS Institute) will be used for all analysis.

\section{Data collection and monitoring}

All data will be collected at the scheduled follow-up times (table 1). Well-trained CRCs examine and enter the data on the case report forms using a specific online database, Electronic Data Capture (Gauss Infomed, Beijing, China). The data manager will ensure that the hard copy of files and electronic data will be preserved properly and timely to control the quality. Only the trial administrator and the statistician have access to the final dataset.

\section{Ethics and dissemination}

This final study protocol, including the final version of the Patient Information and Consent Form, was approved by the ethical committee of the ZOC, Sun Yat-sen University, in April 2019 (ID: 2019KYPJ079). This cohort study publication was written by investigators who approve the submission prior to any publication. Any presentation must be approved by the investigators domestically and internationally.

Contributors XZ and SC had the original idea for the study and designed the project. JBJ was involved in the drafting of the manuscript and critical revision of the study design and protocol. WW and SC designed the qualitative process evaluation and contributed to study management and analysis. LJ contributed to the design of the outcome measures and statistical analysis as senior statistician for the project. YS, WW and SC wrote the primary manuscript together. XZ and FL revised the manuscript. MC, JL and JZ were clinical research coordinators of the project. FL, KG, RZ, WC and JX contributed in collecting the data. All the authors reviewed and approved the final manuscript.

Funding This study was funded by High-level Hospital Construction Project, Zhongshan Ophthalmic Center, Sun Yat-sen University (Funding number: 303020104)

Competing interests JBJ: Patent application: Europäische Patentanmeldung 16720043.5 and Patent application US 20190085065 A1, agents for use in the therapeutic or prophylactic treatment of myopia or hyperopia. 
Patient and public involvement Patients and/or the public were not involved in the design or conduct or reporting or dissemination plans of this research.

Patient consent for publication Not required.

Provenance and peer review Not commissioned; externally peer reviewed.

Open access This is an open access article distributed in accordance with the Creative Commons Attribution Non Commercial (CC BY-NC 4.0) license, which permits others to distribute, remix, adapt, build upon this work non-commercially, and license their derivative works on different terms, provided the original work is properly cited, appropriate credit is given, any changes made indicated, and the use is non-commercial. See: http://creativecommons.org/licenses/by-nc/4.0/.

\section{ORCID iDs}

Wei Wang http://orcid.org/0000-0002-5273-3332

Jost B Jonas http://orcid.org/0000-0003-2972-5227

Xiulan Zhang http://orcid.org/0000-0003-1522-2653

\section{REFERENCES}

1 Flaxman SR, Bourne RRA, Resnikoff S, et al. Global causes of blindness and distance vision impairment 1990-2020: a systematic review and meta-analysis. Lancet Glob Health 2017;5:e1221-34.

2 Xu L, Wang Y, Wang S, et al. High myopia and glaucoma susceptibility the Beijing eye study. Ophthalmology 2007;114:216-20.

3 Bikbov MM, Gilmanshin TR, Kazakbaeva GM, et al. Prevalence of myopic maculopathy among adults in a Russian population. JAMA Netw Open 2020;3:e200567.

4 Jonas JB, Weber P, Nagaoka N, et al. Glaucoma in high myopia and parapapillary delta zone. PLoS One 2017;12:e0175120.

5 Jonas JB, Gusek GC, Naumann GO. Optic disc, cup and neuroretinal rim size, configuration and correlations in normal eyes. Invest Ophthalmol Vis Sci 1988;29:1151-8.

6 Tan NYQ, Sng CCA, Jonas JB, et al. Glaucoma in myopia: diagnostic dilemmas. Br J Ophthalmol 2019;103:1347-55

7 Jonas JB, Nagaoka N, Fang YX, et al. Intraocular pressure and glaucomatous optic neuropathy in high myopia. Invest Ophthalmol Vis Sci 2017:58:5897-906.

8 Choi YJ, Jeoung JW, Park KH, et al. Glaucoma detection ability of ganglion cell-inner plexiform layer thickness by spectral-domain optical coherence tomography in high myopia. Invest Ophthalmol Vis Sci 2013;54:2296-304.

9 Lee K, Maeng KJ, Kim JY, et al. Diagnostic ability of vessel density measured by spectral-domain optical coherence tomography angiography for glaucoma in patients with high myopia. Sci Rep 2020;10:3027.

10 Ang M, Tan ACS, Cheung CMG, et al. Optical coherence tomography angiography: a review of current and future clinical applications. Graefes Arch Clin Exp Ophthalmol 2018;256:237-45.

11 Suwan Y, Fard MA, Geyman LS, et al. Association of myopia with peripapillary perfused capillary density in patients with glaucoma: an optical coherence tomography angiography study. JAMA Ophthalmol 2018;136:507-13.

12 Fang Y, Yokoi T, Nagaoka N, et al. Progression of myopic maculopathy during 18-year follow-up. Ophthalmology 2018;125:863-77.

13 Yan YN, Wang YX, Yang Y, et al. Ten-year progression of myopic maculopathy: the Beijing eye study 2001-2011. Ophthalmology 2018;125:1253-63.

14 Ohno-Matsui K, Kawasaki R, Jonas JB, et al. International photographic classification and grading system for myopic maculopathy. Am J Ophthalmol 2015;159:877-83.

15 Pan C-W, Cheung CY, Aung T, et al. Differential associations of myopia with major age-related eye diseases: the Singapore Indian eye study. Ophthalmology 2013;120:284-91.

16 Prum BE, Rosenberg LF, Gedde SJ, et al. Primary Open-Angle Glaucoma Preferred Practice Pattern(®) Guidelines. Ophthalmology 2016;123:P41-111.

17 Prum BE, Lim MC, Mansberger SL, et al. Primary Open-Angle Glaucoma Suspect Preferred Practice Pattern(®) Guidelines. Ophthalmology 2016;123:P112-51.

18 Ding X, Chang RT, Guo X, et al. Visual field defect classification in the Zhongshan ophthalmic Center-Brien Holden vision Institute high myopia registry study. Br J Ophthalmol 2016;100:1697-702.

19 Garway-Heath DF, Crabb DP, Bunce C, et al. Latanoprost for openangle glaucoma (UKGTS): a randomised, multicentre, placebocontrolled trial. Lancet 2015;385:1295-304.

20 Keltner JL, Johnson CA, Anderson DR, et al. The association between glaucomatous visual fields and optic nerve head features in the ocular hypertension treatment study. Ophthalmology 2006;113:1603-12. 